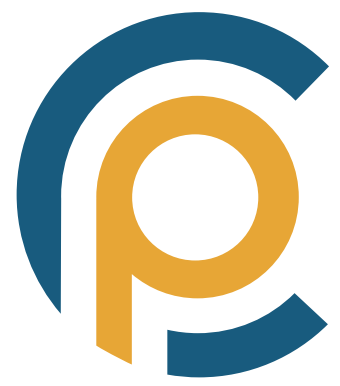

\title{
Transitando la Ruptura Familiar: Una Aproximación Hacia la Coordinación de Parentalidad
}

\author{
Transiting the Family Breakup: \\ An Approach to Parenting Coordination
}

Recibido: 04/03/2020 | Revisado: 01/05/2020 | Aceptado: 21/05/2020 | Publicado: 30/05/2020

\section{Verónica Riquelme Soto}

Universidad de Valencia

veronica.riquelme@uv.es

https://orcid.org/0000-0003-0737-7559

\section{iD Piedad Sahuquillo Mateo}

Universidad de Valencia

piedad.sahuquillo@uv.es

https://orcid.org/0000-0002-8450-2475

\section{Paz Cánovas Leonhardt \\ Universidad de Valencia \\ Paz.canovas@uv.es \\ https://orcid.org/0000-0001-8659-0205}

Resumen: El modelo tradicional de familia ha sufrido cambios durante los últimos tiempos, existiendo un incremento de separaciones y divorcios, a la vez que un aumento en la cifra de los procesos de ruptura judicializados. En torno a dicha realidad socio-familiar, es importante garantizar el derecho fundamental de los menores a relacionarse adecuadamente tanto con su padre como con su madre manteniendo todos sus vínculos. Ello se debe a que el problema surge no por el hecho de que los progenitores decidan poner fin a su vida en común, sino cuando se hace partícipes a sus hijos de los conflictos que ha generado la separación o divorcio.

Dichos procesos de ruptura familiar con alta conflictividad suponen un riesgo importante en lo que refiere a la garantía del bienestar de los niños y niñas involucrados en estos. Son cada vez más los autores que exponen que los procesos de separación o divorcio conflictivos repercuten en los menores, provocando un incremento de problemas de comportamiento, emocionales o psicológicos. La presente investigación tiene por objetivo el desarrollo de un análisis de contenido desde el que poder analizar la literatura científica, nacional e internacional, que se genera en torno a dicha problemática de índole familiar. Los resultados obtenidos vislumbran una primera aproximación que permita profundizar en los procesos de ruptura familiar y conocer el impacto de la evidencia científica en torno a la realidad de la coordinación de parentalidad.

Palabras clave: relaciones familiares, cuidado de la infancia, necesidades de la infancia, relaciones padres-hijos, problemas familiares, conflictos, resolución de conflictos, influencias familiares.
Abstract: The traditional family model has undergone changes in recent times, there has been an increase in separations and divorces, as well as an increase in the number of judicial breakup proceedings subject to high levels of conflict. Regarding this socio-family reality, it is important to guarantee the fundamental right of minors to relate adequately with both their father and their mother, maintaining all their ties. The problem arises when their children are involved in the conflicts generated by the separation or divorce.

These processes of family breakup with high levels of conflict are an important risk in terms of guaranteeing the well-being of the children involved in them. In the same way, there are more and more authors who explain that processes of separation or divorce conflict affect the children in different dimensions, causing an increase in behavioral, emotional or psychological problems. The aim of this study is to develop a content analysis to know and analyze the scientific literature, national and international, around this area. Our results show a first approximation from which to investigate and deepen the family breakup processes and to know the impact of scientific evidence around the reality of parenting coordination.

Keywords: family relationship, child care, childhood needs, parent child relationship, family problems, conflict, conflict resolution, family influence. 
La familia constituye el núcleo social básico de convivencia, siendo fundamentales su estructura y las relaciones que dentro se producen para el desarrollo de cada miembro que la compone. La familia es el primer espacio educativo, no sólo porque precede en el tiempo a cualquier otra instancia educativa, sino también en cuanto a su potencial en la formación de los individuos.

Aquello que ocurra dentro del contexto familiar en los primeros años de vida en los menores, va a tener una influencia decisiva en la vida posterior. Por ello, no se concibe a la familia como una entidad estática, sino que se encuentra en un cambio constante, al igual que sus contextos sociales.

Así, los procesos de ruptura familiar suponen para la familia una serie de cambios y de desafíos en el marco del ciclo vital familiar, debido a las alteraciones que provoca en los roles y funcionamiento de la misma. En España, la ruptura familiar se ha convertido durante los últimos tiempos en una realidad cotidiana cuyo crecimiento es reflejado en las cifras estadísticas disponibles.

Como señala el Instituto Nacional de Estadística (2019, p. 1), los últimos estudios relativos al número de separaciones y divorcios en España indican que durante el año 2018 se produjeron 95.254 divorcios, 4.098 separaciones y 92 nulidades. Los divorcios representaron el 95,8\% del total, las separaciones el $4,1 \%$ y las nulidades el $0,1 \%$ restante.

Dichos datos ponen en evidencia el elevado número de procesos de separaciones y/o divorcios que acontecen dentro del marco español, siendo una realidad que implica la necesidad de contemplar las rupturas familiares como una posible crisis dentro del sistema familiar.

\section{Procesos de Separación o Divorcio Conflictivos}

La ruptura familiar en nuestro país, se ha convertido en los últimos años en una realidad cotidiana cuyo crecimiento es reflejado en las cifras estadísticas disponibles. Asimismo, "según los datos ofrecidos por el Consejo General del Poder Judicial, las separaciones y divorcios desde su regulación en España en 1981, han aumentado de forma constante" (Arch, 2010, p. 115).

En cualquier caso, la separación o divorcio de una pareja constituye una crisis de transición cuyo resultado suele definir una realidad familiar probablemente más compleja, aunque no por ello necesariamente más perjudicial. En palabras de Bolaños (1998):

El conflicto puede ser productivo cuando conduce a una solución creativa que podría haber pasado desapercibida de no existir la disputa. A su vez, el conflicto puede ser funcional cuando provoca la distancia emocional necesaria entre dos individuos dolidos. En cambio, el conflicto es destructivo cuando conlleva tensión prolongada, produce hostilidad crónica, reduce drásticamente el nivel de vida, perjudica el bienestar psicológico o destruye las relaciones familiares.

Ahora bien, se concibe de interés ahondar en dichos procesos de separación o divorcio que derivan de conflictos punitivos, pues son cada vez más los casos de ruptura familiar que se inician bajo un clima de elevado conflicto, llegando a la judicialización de dichos procesos e implicando a los menores en los mismos. En relación a ello, diversos autores señalan (Coates et al., 2004; Arch, 2010; García-Herrera, 2016) que en torno al 10\% de las separaciones y divorcios son calificadas como altamente conflictivas, siendo este tipo de casos los que consumen el 90\% de la atención de los Tribunales. Como expone García-Herrera (2016, p. 18), dicha situación no resulta ajena a nuestro sistema y se confirma a través de las estadísticas del INE en conexión con el Consejo General del Poder Judicial.

Así pues, pese a que en algunas ocasiones es la propia disolución marital la que da lugar a los conflictos entre los progenitores, suele ser más frecuente que estos sean una prolongación de lo que acontecía previamente en la relación de pareja. En estos casos, los divorcios sujetos a dinámicas punitivas se caracterizan, además de por su cronicidad, por disputas legales intratables, conflictos continuos acerca de la crianza de los hijos e hijas, hostilidad, amenazas físicas y violencia intermitente. 
En relación con ello, son muchos los autores (Arch, 2010; Henry et al., 2011; Amato y Anthony, 2014; Avedillo et al., 2015; Fariña et al., 2017; Baker y Eicher, 2016; Quigley y Cyr, 2018) que han estudiado dicho fenómeno, llegando a establecer que entre las principales razones de que los conflictos se centren en aquellos temas vinculados con los menores (en especial los relacionados con el régimen de visitas y la custodia) es que estos representan el único vínculo entre los progenitores.

En ese sentido, en las últimas décadas, un gran número de investigaciones (Ahrons, 2006; Amato, 1993; Cantón, Cortés y Justicia, 2002; Amato y Afifi, 2006; Arch, 2010; Bonasa, 2010; D'Abate, 2016; Gaffal, 2012; Gómez-Ortiz, Martín y Ortega-Ruiz, 2017; Martinón et al., 2017; Amundson y Short, 2018; Quigley y Cyr, 2018) se han interesado en estudiar las implicaciones que el divorcio presenta para los hijos e hijas, llegando a recopilar la mayor parte de éstas numerosas evidencias que sugieren una asociación entre dicha situación y una disminución significativa en sus niveles de bienestar.

Si bien lo ideal es mantener la misma estructura a lo largo de todo el ciclo vital de las familias, lo que más perjudica a los hijos e hijas no es la separación o divorcio en sí, sino la forma en que sus padres transitan dicho proceso. Tal y como presentan Seijo, Fariña y Novo (2002, p. 204), se conoce que los procesos de separación o divorcio conflictivos repercuten en los menores en diferentes dimensiones, provocando un incremento de problemas de comportamiento, emocionales o psicológicos, que implican una disfuncionalidad para muchos de ellos. Y, aunque las consecuencias psico-emocionales quizás sean las más relevantes, también se aprecian a nivel de la salud física. Estudios posteriores (Redondo, Fariña, Seijo, Novo y Arce, 2019) siguen constatando la existencia de sintomatología diversa asociada a los procesos de separación y divorcio, especialmente en alta conflictividad por la guarda y custodia.

Finalmente, se ha de mencionar que las consecuencias más devastadoras son de índole psico-emocional, siendo habitual que después del divorcio los hijos e hijas manifiesten una serie de reacciones tales como: sentimientos de abandono, conflicto de lealtades, sentimientos de impotencia, rechazo y culpabilidad. Todos los sentimientos que surgen en los menores, pueden derivar en conductas regresivas (enuresis, encopresis o chuparse el dedo) o una maduración impropia de su edad, mostrándose excesivamente responsables de lo que acontece en su entorno, sumamente cooperativos, autodisciplinados y autocontrolados.

\section{La Coordinación de Parentalidad}

Los conflictos derivados de la ruptura familiar que asumen dinámicas punitivas para los miembros, no responden a procesos de intervención basados en la mediación ni en intervenciones familiares convencionales. Son cada vez más los autores (Henry et al., 2011; Rodríguez-Domínguez y Carbonell, 2014; Ferraro, Davis, Petren y Pasley, 2016; Bertrand y Boyd, 2017; Amundson y Short, 2018; Quigley y Cyr, 2018; Rodríguez y Soto, 2015) que durante los últimos años han ido desarrollando diversas investigaciones científicas en torno a la Coordinación de Parentalidad como proceso de intervención, siendo su principal objetivo facilitar la comunicación entre los progenitores respecto a las necesidades de los hijos.

La Coordinación de Parentalidad surge a mediados de los años ochenta en el marco contextual de Estados Unidos, llegando a adoptar diferentes denominaciones, tales como: special master, wiseperson, family court advisor, mediator-arbitrator o parenting plan coordinator. A inicios de los años noventa, se presenta la Coordinación de Parentalidad como un proceso de intervención centrado en abordar aquellos casos judicializados que se encuentran sujetos a un elevado nivel de conflictividad, y en el que los hijos se ven inmersos en una realidad de ruptura familiar punitiva.

De esta forma, Quigley y Cyr (2018) indican que la Coordinación de Parentalidad es un proceso alternativo de resolución de conflictos dirigido a progenitores que presentan un elevado conflicto tras el proceso de separación o divorcio y que encuentran problemas a la hora de implementar las órdenes judiciales y los planes de crianza. En relación con ello, se mencionan como pioneros de la Coordina- 
ción de Parentalidad los estados de California y Colorado (Kelly, 2014). A su vez, Fariña et al. (2017, p. 159) indican que Oklahoma en 2001 fue el primer estado en desarrollar una normativa legal para dar soporte a dichos profesionales, siguiéndole rápidamente otros estados, con el objeto de conferirle una regulación estatutaria ad hoc.

Del mismo modo, la coyuntura en la que surge la figura del coordinador de parentalidad, se caracteriza por un elevado número de divorcios, una creciente demanda de la custodia compartida y el uso principal de la vía judicial, por parte de algunos progenitores, para solventar las cuestiones relativas al plan de parentalidad, a lo que había que añadir que las sentencias judiciales no resolvían el problema, produciéndose una judicialización de la relación parental, acompañado del consecuente daño a los hijos e hijas.

A partir de diferentes estudios (Coates et al., 2004; Vareschi y Bursik, 2005; Henry et al., 2011; Nielsen, 2011; Baker y Eicher, 2016; D’Abate, 2016; Ferraro, Davis, Petren y Pasley, 2016; Bertrand y Boyd, 2017; Berryhill, 2017; Gürmen et al., 2017; Marinho, 2017; Amundson y Short, 2018; Quigley y Cyr, 2018), se estima que entre el $8 \%$ y el $12 \%$ de los progenitores que ya se encuentran separados o divorciados, continúan con un nivel alto de conflicto tras la ruptura familiar. Dichas realidades hacen alusión a aquellas situaciones conflictivas que se cronifican en el tiempo y que se caracterizan por falta de confianza entre los progenitores, la incapacidad para comunicar sus sentimientos y necesidades de manera apropiada y un uso frecuente del sistema judicial.

En el sistema judicial, las familias con un alto conflicto generado durante el proceso de ruptura familiar son aquellas cuyos progenitores presentan una gran dificultad a la hora de resolver diferencias, especialmente en lo que respecta a la custodia de los hijos, las visitas y la crianza de los mismos. En este sentido, a lo largo de las últimas décadas, un gran número de investigaciones (Arch, 2010; Bonasa, 2010; Amato y Anthony, 2014; Kelly, 2014; Baker y Eichler, 2016; Fariña et al., 2017; Martinón et al., 2017; Amundson y Short, 2018; Quigley y Cyr, 2018) se han centrado en estudiar las implicaciones que el divorcio presenta para los hijos e hijas, llegando a recopilar numerosas evidencias que sugieren una relación entre dicha realidad de ruptura familiar y una disminución significativa en sus niveles de bienestar.

Asimismo, los menores que conviven bajo constantes conflictos entre sus progenitores tienden a desarrollar intensos sentimientos de ansiedad, angustia e ira, poniendo en perjuicio su correcto desarrollo bio-psico-social. En palabras de Quigley y Cyr (2018, p. 501), décadas de investigación señalan que los conflictos entre los padres y madres son uno de los principales factores de riesgo en la adaptación de los niños y adolescentes después de la separación de los padres.

La Coordinación de Parentalidad permite a los progenitores aprender a desvincularse uno del otro, generando nuevos modelos relacionales que les ayude a adoptar habilidades de comunicación asertivas y no violentas, a la vez que favorece el bienestar de los menores.

Finalmente, se estima relevante hacer especial mención a la figura de los coordinadores de parentalidad en lo que respecta a la implementación de Programas de Coordinación de Parentalidad (Rodríguez- Domínguez y Carbonell, 2014; Cyr, Macé y Quigley, 2016; Bertrand y Boyd, 2017; Fariña et al., 2017), ya que a través de los estudios sobre buenas prácticas, se evidencian las múltiples ventajas en la resolución alternativa de conflictos en los tribunales de familia como forma de gestión intensiva, junto con los casos de custodia de menores cuyos progenitores permanecían atrapados en conflictos crónicos.

En suma, la función esencial de dichos programas recae en la creación de planes adecuados para construir relaciones parentales duraderas, basadas en la resolución de conflictos a través de la comunicación no violenta, permitiendo promover una parentalidad positiva. 


\section{Objetivo General}

Conocer la evidencia científica existente, tanto en el panorama nacional como internacional, en relación con los procesos de ruptura familiar conflictivos y la Coordinación de Parentalidad.

\section{Objetivos Específicos}

, Conocer la realidad de las familias que se encuentran expuestas a constantes conflictos punitivos generados por procesos de separación o divorcio.

> Analizar el perfil de familias que acuden al servicio de Coordinación de Parentalidad.

, Identificar los principales conflictos que presentan las familias que viven procesos de separación o divorcio sujetos a constantes litigios.

> Desarrollar una aproximación en torno a la Coordinación de Parentalidad comprendida como proceso de intervención.

Método

Para desarrollar un trabajo de investigación se concibe necesario llevar a cabo un procedimiento metódico que permita la adecuada consecución de los objetivos propuestos, así como una formulación clara, concreta y precisa del problema, junto con una metodología de investigación rigurosa y adecuada al tipo de estudio.

El presente estudio se ubica dentro de un modelo de investigación propio al paradigma cualitativo, puesto que se centra en aspectos descriptivos, especialmente a través del análisis de contenido (método para estudiar y analizar las comunicaciones de una forma sistemática, objetiva y cuantitativa).

En la actualidad, se puede afirmar que el análisis de contenido ha llegado a ser un método científico capaz de ofrecer inferencias a partir de datos esencialmente verbales, simbólicos o comunicativos. Para ello es necesario delimitar el objeto de estudio y el cómo se va analizar, de manera que durante el transcurso de la investigación se dé respuesta a las preguntas por las cuales se genera el estudio en cuestión.

Así pues, el análisis de contenido se basa en la lectura como instrumento de recogida de información, realizándose bajo el método científico, puesto que debe ser sistemático, objetivo, replicable y valido.

Por consiguiente, el presente estudio concibe el análisis de contenido como el método desde el que se permite estudiar qué tipos de contenidos manifiestos existen en una comunicación escrita, clasificando sus diferentes partes de acuerdo con unas categorías previamente establecidas, por lo que existe la posibilidad de identificarlas de manera sistemática y objetiva dentro de un mensaje. Para ello es necesario delimitar el objeto de estudio y el cómo se va analizar, de manera que durante el transcurso de la investigación se dé respuesta a las preguntas por las cuales se genera el estudio en cuestión. Dicha investigación, se centra en conocer el estado de la cuestión respecto a las familias que se encuentran inmersas en procesos de separación o divorcio conflictivos, junto con aquellas intervenciones basadas en la Coordinación de Parentalidad desde las que se abordan dichas realidades familiares. 
Resultados

\section{Fase 1: Definición del Universo}

Toda investigación científica debe definir el campo de estudio como primera tarea, pues es de suma importancia concretar el qué se va a investigar. La definición del universo estudiado marca la dirección y el camino a seguir durante el trascurso de la propia investigación. En nuestro caso, el universo objeto de estudio queda configurado por la selección bibliográfica examinada, desde la que se lleva a cabo la revisión del estado de la cuestión relativa a los procesos de separación o divorcio conflictivos y la intervención que se realiza a través de los servicios de Coordinación de Parentalidad en dichos casos.

\section{Fase 2: Determinar los Objetivos}

Los objetivos específicos relativos al análisis de contenido como metodología de investigación han quedado ya explicitados en el apartado 2.2. del presente trabajo. Cabe señalar que la determinación de los mismos se lleva a cabo a partir de una aproximación bibliográfica en torno al estado de la cuestión de los procesos de separación o divorcio conflictivos, y su intervención desarrollada a través de los servicios de Coordinación de Parentalidad, desde el que se pretende:

> Establecer indicadores o unidades de análisis tras la revisión documental.

, Desarrollar la codificación de las unidades de análisis.

, Establecer categorías de análisis.

\section{Fase 3: Elección de Documentos}

La selección de documentos e información resulta, quizás, la fase más importante dentro del marco de la presente investigación, puesto que a través de esta se pretende conocer la realidad de las familias que se encuentran sujetas a procesos de ruptura familiar conflictivos, junto con la aplicación de la Coordinación de Parentalidad las familias que responden a dicho perfil.

Es por ello que la selección de los documentos conforma el cuerpo central del estudio, permitiendo indagar en el panorama nacional e internacional sobre el impacto de dicha figura profesional y su eficacia en aquellos casos en los que las familias se encuentran sometidas a constantes litigios, en los que los menores forman parte, y en ocasiones son empleados como medio de transacción entre los progenitores enfrentados.

El desarrollo de dicha fase, se lleva a cabo a partir de un proceso de selección riguroso guiado por el tema objeto de estudio, tal y como se refleja en la figura 1. De este modo, se hace uso de diferentes fuentes de información desde las que poder abordar de forma eficaz el estado de la cuestión propio a las rupturas familiares conflictivas y su consecutiva intervención. Las fuentes de información seleccionadas se encuentran expuestas en la tabla 1. 


\section{Figura 1}

Desarrollo del proceso de selección de las fuentes de información

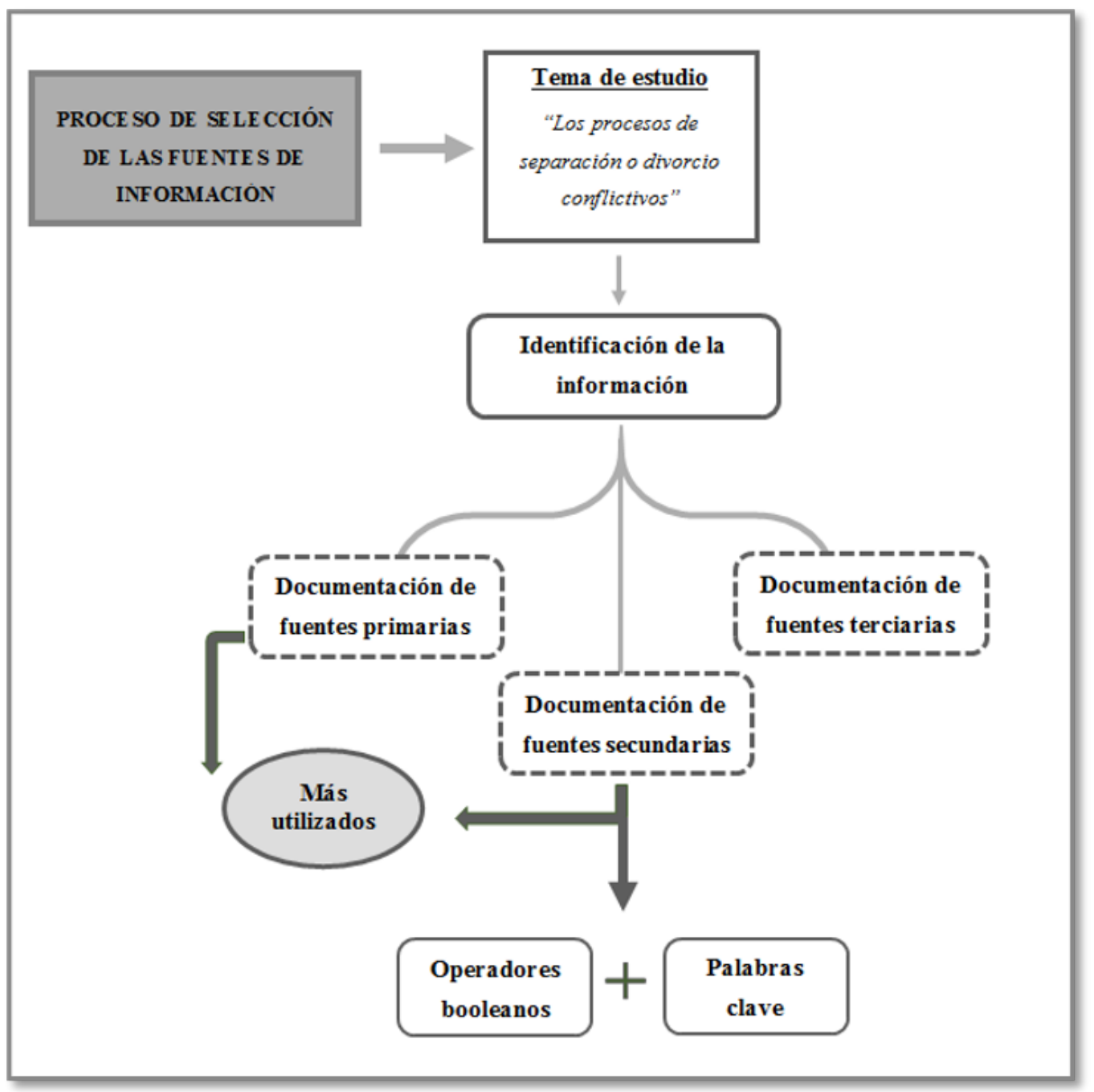

Fuente: Elaboración propia

Tabla 1

Tipos de documentación empleadas en el proceso del análisis de contenido

\begin{tabular}{|l|l|}
\hline Documentación primaria & Libros, proyectos de investigación y revistas. \\
\hline Documentación secundaria & Catálogos y bases de datos. \\
\hline Documentación terciaria & Metabuscadores. \\
\hline
\end{tabular}

Fuente: Elaboración propia

Del mismo modo, se estima oportuno señalar que para la selección documental, se establecieron diferentes criterios de búsqueda desde los delimitar el material científico recabado. Estos son los siguientes:

> Los estudios revisados abordan la realidad de las familias en procesos de separación y/o divorcio conflictivo.

> Las investigaciones han de presentar los servicios de la Coordinación de Parentalidad en relación a las familias que se encuentran inmersas en procesos de ruptura familiar sujetos a un elevado conflicto. 
, Los estudios indican las consecuencias que tienen los procesos de separación o divorcio conflictivos en los hijos e hijas.

> El material revisado presenta buenos índices de calidad respecto al rigor científico.

, Pertenecen tanto a los estudios cualitativos como cuantitativos.

, Escritos en inglés y en español.

> Publicaciones con menos, o igual, de cinco años (2014-2018), a excepción de material relacionado con los estudios pioneros o de referencia en cuanto al tema de estudio.

Ahora bien, entre los principales materiales seleccionados se encuentran libros e investigaciones realizadas por autores de gran influencia en el ámbito de estudio, tanto en el marco nacional como internacional. El empleo de los operadores booleanos y las palabras clave para la búsqueda y selección de la información, resultó de gran interés debido a que a través de estos se reconocieron diferentes unidades de análisis que fueron extraídas en la siguiente fase del análisis de contenido.

Del mismo modo, se estima relevante señalar que entre las principales bases de datos seleccionadas para el desarrollo de la presente fase se encuentran las siguientes: Eric, Journal Citation Reports, Scopus, Dialnet, Teseo, Redalic, Web os Science, Research Gate y Taylor \& Francis online.

\section{Fase 4: Elaboración de Indicadores o Unidades de Análisis}

La elaboración de los indicadores o unidades de análisis, son de gran relevancia para la elaboración del análisis de contenido. A través de dichos indicadores, se lleva a cabo una primera aproximación a los elementos de mayor interés para el estudio, presentes en la documentación seleccionada. Entre los indicadores seleccionados se encuentran:

, Unidades genéricas: Dichas unidades hacen alusión a las diversas partes de la realidad documental sometida a análisis que el investigador considera como separadas e independientes entre sí.

> Unidades de registro:Para efectos de este estudio, se han identificado 37 unidades de registro, de las cuales se extraen diferentes elementos de análisis. Las unidades de registro generadas para el correcto análisis de contenido son:

- Proceso de separación o divorcio contencioso.

- Impacto en los menores de la exposición a los conflictos generados por la separación o divorcio.

- Proceso de intervención basado en la Coordinación de Parentalidad.

, Unidades de contexto: Documentación científica de autores y ejemplos de buenas prácticas en el marco nacional e internacional.

A continuación, en la tabla 2, se presentan los principales indicadores establecidos tras la revisión documental, ya que a través de esta se exponen aquellos elementos compartidos en cada uno de los materiales revisados, al igual que se aprecian diferentes unidades de análisis emergentes respecto a las consecuencias de los menores tras la ruptura familiar conflictiva. 


\section{Tabla 2}

Desarrollo de indicadores a través de las unidades de registro

\begin{tabular}{|c|c|}
\hline Unidades de registro & Indicadores - Unidades de análisis \\
\hline \multirow{7}{*}{ Ruptura familiar } & $\begin{array}{l}\text { Problemas adversos en términos socioeconómicos: } \\
\text {, Umbral de pobreza. } \\
\text {, Manutención de los hijos e hijas. }\end{array}$ \\
\hline & $\begin{array}{l}\text { Problemas adversos en términos de ajuste psicológico: } \\
\text {, Gestión del estrés. } \\
\text {, Depresión. }\end{array}$ \\
\hline & $\begin{array}{l}\text { La forma en la que se gestiones el proceso de separación o divorcio } \\
\text { podrá ocasionar afectación negativa en los miembros, o positiva. }\end{array}$ \\
\hline & $\begin{array}{l}\text { El proceso judicial en España para la separación o divorcio, tiende a } \\
\text { agudizar los conflictos familiares. }\end{array}$ \\
\hline & $\begin{array}{l}\text { Los conflictos familiares derivados de la ruptura familiar pueden llegar } \\
\text { a cronificarse en el tiempo. }\end{array}$ \\
\hline & $\begin{array}{l}\text { Conflictos generados, en gran parte, por: } \\
\text { > Custodia de los hijos. } \\
\text { > Régimen de visitas. } \\
\text { > La relación de los progenitores con los hijos. } \\
\text {, La relación con las familias extensas. } \\
\text {, Estilos educativos no compartidos entre los progenitores. }\end{array}$ \\
\hline & $\begin{array}{l}\text { Los progenitores tienden a hacer partícipes a los hijos en los conflictos } \\
\text { derivados de la ruptura familiar. }\end{array}$ \\
\hline \multirow{5}{*}{$\begin{array}{l}\text { Impacto en los menores de la } \\
\text { exposición a los conflictos generados } \\
\text { por la separación o divorcio }\end{array}$} & Riesgo de problemas de ajuste emocional y conductual. \\
\hline & $\begin{array}{l}\text { Aumento del riesgo de padecer enfermedades crónicas agudas, } \\
\text { obesidad, hipertensión, enfermedades coronarias y alteraciones } \\
\text { psicosomáticas. }\end{array}$ \\
\hline & Aumento de sintomatología propia al Estrés Postraumático. \\
\hline & Exposición a prácticas alienadoras familiares. \\
\hline & $\begin{array}{l}\text { Presentan dificultades durante el proceso de separación o divorcio en } \\
\text { la escuela. }\end{array}$ \\
\hline \multirow{6}{*}{ La Coordinación de Parentalidad } & Nace en Canadá y en Estados Unidos. \\
\hline & Su figura presenta un perfil propio a la psicología forense. \\
\hline & $\begin{array}{l}\text { El principio rector en las intervenciones es garantizar el bienestar de } \\
\text { los menores. }\end{array}$ \\
\hline & $\begin{array}{l}\text { Su objetivo es generar nuevos modelos relacionales entre los } \\
\text { progenitores que les ayude a adoptar habilidades de comunicación } \\
\text { asertivas y no violentas, a la vez que favorece el bienestar de los } \\
\text { menores. }\end{array}$ \\
\hline & $\begin{array}{l}\text { Los procesos de intervención se encuentran orientados a la resolución } \\
\text { pacífica de conflictos, centrada en la comunicación no violenta. }\end{array}$ \\
\hline & $\begin{array}{l}\text { No intervendrá en los casos donde se mantengan activas problemáticas } \\
\text { como: } \\
\text {, El consumo de sustancias tóxicas en alguno de los progenitores. } \\
\text {, Existan pruebas de que continúa la violencia en el hogar. } \\
\text {, Exista maltrato infantil o abuso sexual en los hijos. } \\
\text {, Existan prácticas alienadoras familiares. }\end{array}$ \\
\hline
\end{tabular}

Fuente: Elaboración propia 


\section{Fase 5: Reglas de Numeración o Recuento}

La presente fase hace referencia a la forma de contar las unidades de registro, debido a la importancia de generar un adecuado examen de la realidad documental investigada. De esta forma, los criterios establecidos fueron los siguientes:

- Alusión a las familias que se encuentran sujetas a procesos de separación o divorcio conflictivo.

, La frecuencia: Dicho criterio alude al número de veces que aparecen las unidades de registro.

Ambas son contempladas en el proceso de la creación de categorías, siguiendo como base los indicadores seleccionados en la fase anterior.

\section{Fase 6: La Categorización}

La fase denominada como categorización, consiste en clasificar los elementos de un conjunto a partir de ciertos criterios previamente definidos. La categorización no es una tarea de investigación mecánica, ya que los términos pueden no hacer referencia a los significados que a primera vista expresan o manifiestan, sino estar fuertemente matizados por el contexto. Así pues, en la tabla 3 se presentan las diferentes categorías definidas en función de los indicadores extraídos en la fase expuesta anteriormente.

\section{Tabla 3}

\section{Categorías e indicadores}

\begin{tabular}{|c|c|c|}
\hline Categorías & Indicadores & Frecuencia \\
\hline \multirow{7}{*}{$\begin{array}{l}\text { Proceso de separación o } \\
\text { divorcio contencioso }\end{array}$} & Problemas socioeconómicos & 10 \\
\hline & Problemas de ajuste psicológico & 30 \\
\hline & Gestión de la ruptura familiar y los conflictos derivados de la misma & 34 \\
\hline & $\begin{array}{l}\text { Los procesos de separación o divorcio en el marco nacional } \\
\text { (España) e internacional }\end{array}$ & 21 \\
\hline & Cronificación de los conflictos tras la separación o divorcio & 18 \\
\hline & Principales conflictos tras la ruptura familiar & 34 \\
\hline & Participación del menor en los conflictos parentales & 20 \\
\hline \multirow{5}{*}{$\begin{array}{l}\text { Impacto en los menores } \\
\text { tras la exposición a los } \\
\text { conflictos familiares }\end{array}$} & Problemas emocionales y conductuales & 28 \\
\hline & Problemas de salud & 20 \\
\hline & Sintomatologías de Estrés Postraumático & 9 \\
\hline & Las prácticas alienadoras familiares como factor de riesgo & 12 \\
\hline & Dificultades académicas & 19 \\
\hline \multirow{5}{*}{$\begin{array}{l}\text { La Coordinación de } \\
\text { Parentalidad }\end{array}$} & Origen canadiense y estadounidense & 5 \\
\hline & Figura profesional asociada a la psicología forense & 9 \\
\hline & El objetivo profesional es salvaguardar el bienestar de los menores & 12 \\
\hline & Procesos de intervención centrados en la comunicación no violenta & 12 \\
\hline & $\begin{array}{l}\text { Realidades familiares en las que no se aplica la Coordinación de } \\
\text { Parentalidad }\end{array}$ & 3 \\
\hline
\end{tabular}

Fuente: Elaboración propia 
Cada vez son más las familias que se encuentran sujetas a procesos de separación o divorcio en España, siendo a su vez una realidad que no difiere mucho de otros marcos contextuales internacionales.

Es por ello que tras la revisión documental realizada a través del análisis de contenido, se han extraído los principales temas abordados en la literatura científica respecto a los procesos de separación o divorcio conflictivos, junto con el trabajo realizado con dichas familias en los servicios de Coordinación de Parentalidad. Así pues, podemos afirmar que se han alcanzado los objetivos planteados para este trabajo.

En primer lugar, cabe indicar que existe un elevado número de documentación en relación al proceso de separación o divorcio, siendo menos frecuente encontrarse literatura científica cuando dichos procesos son catalogados como conflictivos o punitivos. Pese a ello, tal y como se refleja en los indicadores del análisis de contenido, junto con sus consecutivas categorías, son cada vez más los estudios que comienzan a investigar dichas realidades familiares, al igual que la implicación y afectación de los menores ante la exposición a los conflictos en cuestión.

En segundo lugar, en la aproximación realizada al objeto de estudio, se vislumbran una ausencia de protocolos de actuación antes el perfil de las familias que se encuentran bajo un elevado nivel de conflictividad debido al proceso de separación o divorcio. De esta manera, se concibe que en el ámbito internacional, el número de investigaciones y estudios en torno a la ruptura familiar conflictiva y a la Coordinación de Parentalidad son mucho más frecuentes, puesto que al ser el lugar de origen de dicho proceso de intervención, existe una mayor evidencia científica en torno al objeto de estudio.

Finalmente, se ha de mencionar la necesidad de profundizar en el impacto que tienen dicha realidad familiar en los menores, dada la importancia de conocer todos aquellos factores de riesgo que pueden afectar a los mismos, incidiendo es su correcto desarrollo bio-psico-social.

\section{Referencias}

Ahrons, C. R. (2006). Family Ties after Divorce: Long-term Implications for Children. Family Process, 46 (1), 53-65. https://doi.org/10.1111/j.1545-5300.2006.00191.x

Amato, P. R. (1993). Children's Adjustment to Divorce: Theories, Hypotheses, and Empirical Support. Journal of Marriage and the Family, 55 (1), 23-38. https://doi.org/10.2307/352954

Amato, P. R. y Afifi, T. D. (2006). Feeling Caught Between Parents: Adult Children's Relation with Parents and Subjective Well-being. Journal of Marriage and Family, 68 (1), 225-235. https://doi. org/10.1111/j.1741-3737.2006.00243.x

Amato, P. R. y Anthony, C. J. (2014). Estimating the Effects of Parental Divorce and Death with fixe Effects Models. Journal of Marriage and Family, 76 (2), 370-386. https://bit.ly/2tX6nd4

Amundson, J. K. y Short, J. (2018). Risk Regulation in High-Conflict Parenting. Journal of Divorce \& Remarriage, 59 (6), 528-537. https://bit.ly/2pmew8c

Arch, M. (2010). Divorcio, Conflicto y Consecuencias en los Hijos: Implicaciones para las Recomendaciones de Guarda y Custodia. Papeles del Psicólogo, 31, 183-190. https://goo.gl/dqsqgL

Avedillo, M., Carrasco, L., Guitart, E. y Sacasas, M. (2015). La Coordinación de Parentalidad: Cuando las Familias ya no Saben qué Hacer. Huygens.

Baker, A. y Eichler, A. (2016). The Linkage Between Parental Alienation Behaviors and Child Alienation. Journal of Divorce \& Remarriage, 57 (7), 475-484. https://doi.org/10.1080/10502556.2016.1220 285 
Berryhill, M. B. (2017). Coparenting and Parental School Involvement. Kansas State University. https:// bit.ly/2tX09Ko

Bertrand, L. D. y Boyd, J. P. (2017). The Development of Parenting Coordination and an Examination of Policies and Practices in Ontario, British Columbia and Alberta. Canadian Research Institute for Law and the Family. http://dx.doi.org/10.11575/PRISM/34535

Bolaños, I. (1998). Conflicto familiar y ruptura matrimonial. Aspectos psicolegales. En J.L. Marrero (Comp.). Psicología Jurídica de la familia (pp. 43-76). Fundación Universidad Empresa, Retos Jurídicos en las Ciencias Sociales.

Bonasa, P. (Coord.). (2010). Procesos disfuncionales en procedimientos de separación y divorcio. ASEMIP.

Cantón, J., Cortés, M. R. y Justicia, M. D. (2002). Las consecuencias del divorcio en los hijos. Psicopatología Clínica, Legal y Forense, 2 (3), 47-66. https://bit.ly/2MedODm

Coates, C. A., Deutsch, R., Starnes, H. H., Sullivan, M. J. y Sydlik, B. (2004). Parenting Coordination for High-conflict Families. Family Court Review, 42 (2), 246-262. https://doi. org/10.1111/j.174-1617.2004.tb00647.x

Cyr, F., Macé, C. y Quigley, C. (2016). Parenting coordination pilot project at the Montreal Superior Court: Implementation and outcome study. Ministry of Justice Montreal.

D'Abate, D. (2016). Use of Solution-focused and Family Narrative Approaches in Working with High Conflict Families: Strategies and Techniques that Can be Utilized in Parenting Coordination and Co-parenting Coaching. Journal of Child Custody, 13 (4), 269-288. https://doi.org/10.1080/1537 9418.2016.1247308

Fariña, F., Pérez-Laboz, V., Vázquez, M. J. y Seijo, D. (2017). Clima familiar y coparentalidad en familias con ruptura de pareja. Revista de estudios e investigación en psicología y educación, 5, 289-296. https://doi.org/10.17979/reipe.2017.0.05.2782

Ferraro, A. J., Davis, T. R., Petren, R. E. y Pasley, K. (2016). Postdivorce Parenting: A Study of Recently Divorced Mothers and Fathers. Journal of Divorce \& Remarriage, 57 (7), 485-503. https://doi.org/ 10.1080/10502556.2016.1220302

Gaffal. M. (2012). Parental Alienation in Divorce Judgments. InDret, 4 (6), 2-23. https://goo.gl/co4x1X

García-Herrera, A. (2016). Reestructuración de la Familia tras la Separación Parental: Mediación Intrajudicial, Mediación en el Punto de Encuentro Familiar y Coordinación de Parentalidad. https://bit. ly/2NWqinQ

Gómez-Ortiz, O., Martín, L. y Ortega-Ruiz, R. (2017). Conflictividad parental, divorcio y ansiedad infantil. Pensamiento Psicológico, 15 (2), 67-78. https://doi.org/10.11144/javerianacali.ppsi15-2.cpda

Gürmen, M. S., Scott, H, C., Brown, E., Orbuch, T. L. y Birditt, S. K. (2017). Divorced Yet Still Together: Ongoing Personal Relationship and Coparenting Among Divorced Parents. Journal of Divorce \& Remarriage, 58 (8), 645-660. https://doi.org/10.1080/10502556.2017.1355172

Henry, W. J., Fieldstone, L., Thompson, M. y Treharne, K. (2011). Parenting Coordination as an Antidote for High-Conflict Divorce and Court Relitigation. Journal of Divorce \& Remarriage, 52 (7), 455-471. https://doi.org/10.1080/10502556.2011.609421

Instituto Nacional de Estadística. (2019). Separación y divorcio. https://bit.ly/3dmbKHK 
Kelly, J.B. (2014). Origins and development of parenting coordination. En S.A. Higuchi y S.J. Lally (Eds.). Parenting coordination in postseparation disputes. American Psychological Association. https:// doi.org/10.1037/14390-002

Marinho, S. (2017). Separate Mothering and Fathering: The Plurality of Parenting Within the Framework of Postdivorce Shared Parenting Norms. Journal of Divorce \& Remarriage, 58 (4), 288-309. https://doi.org/10.1080/10502556.2017.1305852

Martinón, J. M., Fariña, F., Corras, T., Seijo, D., Soto, A. y Novo, M. (2017). Impacto de la Ruptura de los Progenitores en el Estado de Salud Física de los Hijos. European Journal of Education and Psychology, 10 (1), 9-14. https://doi.org/10.1016/j.ejeps.2016.10.002

Nielsen, L. (2011). Shared Parenting After Divorce: A Review of Shared Residential Parenting Research. Journal of Divorce \& Remarriage, 52 (8), 586-609. https://doi.org/10.1080/10502556.2011.6199 13

Quigley, C. y Cyr, F. (2018). The Voice of the Child in Parenting Coordination: Views of Children, Parents, and Parenting Coordinators. Journal of Divorce \& Remarriage, 59 (6), 501-527. https://doi.org/10 $.1080 / 10502556.2017 .1403823$

Redondo, L., Fariña, F., Seijó, D., Novo, M. y Arce, R. (2019). Una revisión meta-analítica de las respuestas en escalas clínicas y reestructuradas del MMPI-2/MMPI-2-RF de padres en disputa por la custodia. Anales de psicología, 35, 1, 156-165. https://doi.org/10.6018/analesps.35.1.338381

Rodríguez-Domínguez, C. y Carbonell, X. (2014). The Parenting coordinator: A New Professional Role For The Forensic Psychologist. Papeles del Psicólogo, 35(3), 193-200. https://bit.ly/2QFbt7I

Rodríguez, M. D. y Soto, R. (2015). El coordinador de parentalidad. Una propuesta desde dentro. Psicopatología Clínica, Legal y Forense, 15, 171-187. https://bit.ly/2MPeYoW

Seijo, D., Fariña, F. y Novo, M. (2002). Repercusiones del Proceso de Separación y Divorcio. Recomendaciones Programáticas Para la Intervención con Menores y Progenitores desde el Ámbito Escolar y la administración de Justicia. Publicaciones, 32, 199-218. https://bit.ly/2OGCbLR

Vareschi, C. G. y Bursik, K. (2005). Attachment Style Differences in the Parental Interactions and Adaptation Patterns of Divorcing Parents. Journal of Divorce \& Remarriage, 42 (3), 15-32. https://doi. org/10.1300/j087v42n03_02 